\title{
Simultaneous Perforation of Three Major Liver Blood Vessels by Percutaneous Transhepatic Biliary Drainage
}

Biliovenous fistula represents the most common source of bleeding after percutaneous transhepatic biliary drainage (PTBD), and the course is usually self-limiting [1]. Severe hemobilia from arteriobiliary fistula must be expected in $0.4 \%$ [2] to $2 \%$ [3] of all procedures performed and often necessitates immediate treatment by selective arterial embolization [2] or transbiliary embolization [4]. We report here the simultaneous perforation of a lobar hepatic artery, a lobar portal vein, and the central right portal vein during PTBD for choledocholithiasis. A 76year-old woman with a history of cholecystectomy and Billroth II gastrectomy was admitted to our institution because of multiple bile duct stones and a common bile duct stricture, diagnosed by computed tomography (CT) scan and endoscopic retrograde cholangiography. PTBD was done using a right intercostal approach, revealing massive dilatation of intrahepatic bile ducts and several large calculi on top of a high-grade common bile duct stenosis. After a Terumo wire had been passed through the stricture, a $10-F r$ pigtail drainage catheter was successfully placed in the duodenum. When the pigtail catheter was removed for further tract dilation, 7 days later, massive bleeding occurred from the percutaneous tract, which was controlled by deep placement of a 12-Fr Nimura catheter without sideholes. Selective hepatic arteriography was immediately performed, and showed normal hepatic artery branches without arteriobiliary fistula. However, a portobiliary fistula within S8 and a centrally located lesion of the right por-

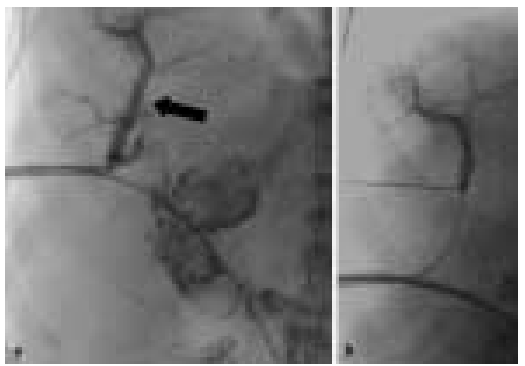

Figure 1 a PTCB revealing massively dilated bile ducts with intraluminal blood coagula. Retrograde tracing of the S8 lobar portal vein (arrow) on retraction of the pigtail catheter is shown. b Percutaneous transhepatic embolization of the perforated lobar portal vein. tal vein were suspected. The portobiliary fistula was verified by retraction of the PTBD device (Figure $1 \mathbf{a}$ ) and the perforated vessel was embolized using a direct transhepatic approach (Figure $\mathbf{1} \mathbf{b}$ ). Since the right portal vein lesion could not be reached via this approach, direct portography was performed, confirming circumferential extravasation of contrast medium from the right portal vein close to the bifurcation (Figure 2), and a Yomed Covered Stent (18 mm length, $10 \mathrm{~mm}$ central and $8 \mathrm{~mm}$ peripheral diameter) was placed to cover the lesion. After a second PTBD device had successfully been placed, via S6, 4 days later, an attempt was being made to remove the Nimura drainage catheter within the first tract, when once again, there was massive bleeding of sudden onset from the percutaneous tract. Retrograde tracing now revealed an arteriobiliary fistula within S8 (Figure 3a). Selective arteriography was performed and the perforated hepatic artery branch was embolized by the application of endocoils (Figure $\mathbf{3 b}$ ). No more bleeding was observed, the Nimura catheter was removed, and further therapy was successfully performed by percutaneous cholangioscopy and laser lithotripsy, after dilation of the second tract. This report describes, for the first time to our knowledge, recurrent serious bleeding episodes after PTBD, from three different lesions of major hepatic blood vessels including the hepatic artery and portal vein. We conclude that severe bleeding complications, with delayed onset and from multiple locations, may occur with PTBD, and that adequate interventional angiography fa-

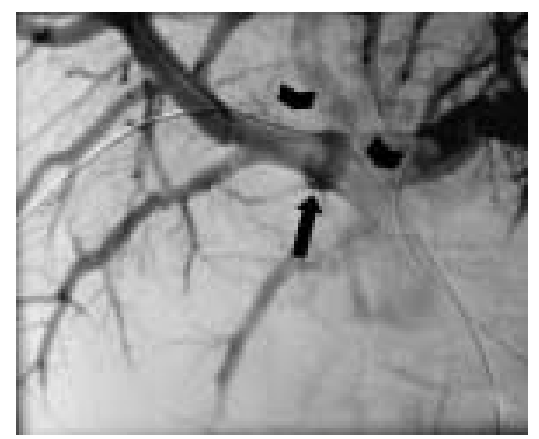

Figure 2 Direct portography shows circumferential extravasation of contrast medium from the right portal vein (arrow). Note the percutaneous transhepatic biliary drainage (PTBD) tract (arrowheads) in the immediate vicinity. cilities should be available when PTBD is carried out.

\section{R. Fritsch ${ }^{1}$, M. Storr ${ }^{1}$, F. Geisler ${ }^{1}$,}

M. Kurjak ${ }^{1}$, H. Berger ${ }^{2}$, T. Rösch ${ }^{1}$,

H. D. Allescher ${ }^{1}$, M. Classen ${ }^{1}$

${ }^{1}$ Department of Internal Medicine II

2 Department of Interventional Radiology, Technical University of Munich, Munich, Germany

\section{References}

${ }^{1}$ Carrasco $\mathrm{CH}$, Zornoza J, Bechtel WJ. Malignant biliary obstruction: complications of percutaneous biliary drainage. Radiology 1984; 152: $343-346$

2 Born P, Golder W, Allescher HD et al. Severe arterial bleeding after percutaneous transhepatic cholangiographic drainage. Endoscopy 1995; 27: 343

${ }^{3}$ L'Hermine C, Ernst O, Delemazure O, Sergent $\mathrm{G}$. Arterial complications of percutaneous transhepatic biliary drainage. Cardiovasc Intervent Radiol 1996; 19: 160-164

${ }^{4}$ Nakagawa N, Nakajima Y, Bird SM, Wakabayashi M. Immediate transbiliary embolization of a biliary-hepatic artery fistula encountered during access for percutaneous biliary drainage. Cardiovasc Intervent Radiol 1994; 17: 295-297

\section{Corresponding Author}

\section{Storr, M.D.}

Department of Internal Medicine II Technical University of Munich Ismaninger Str. 22

81675 München, Germany

E-mail: Martin.Storr@lrz.tumuenchen.de

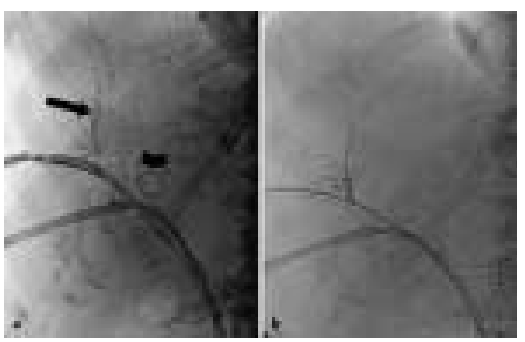

Figure 3 a Retrograde tracing via PTBD revealing arteriobiliary fistula within S8 (arrow). The covered stent (arrowhead) which had been placed in the right portal vein is in situ. $\mathbf{b}$ Selective angiography image of S8 hepatic artery branch before successful application of endocoils (not shown). 